\title{
Postal reminders can improve attendance at orthodontic clinics
}

\author{
Do postal reminders reduce non-attendance in patients invited to an \\ orthodontic clinic?
}

Can S, Macfarlane T, O'Brien KD. The use of postal reminders to reduce non-attendance at an orthodontic clinic: $A$ randomised controlled trial. Br Dent J 2003: 195:199-201

Design A randomised controlled trial in an NHS hospital orthodontic clinic.

Intervention Participants either received an appointment or an appointment plus a reminder at least 2 weeks before their appointment. The reminder letter was sent with a stamped addressed postcard, which the patient was asked to return, to confirm the appointment.

Outcome measure Patient attendance at the clinic.

Results A total of 231 patients were entered into the study. Patients who received a reminder and returned the confirmation were significantly less likely to fail the appointment than those who did not receive a reminder (see Table 1 ). $27 \%$ of girls failed appointments compared with $15 \%$ of boys. Those from areas of high social deprivation were more likely to fail to attend appointment than people who were more affluent.

Conclusions The use of postal reminders for orthodontic consultation appointments appears to result in a useful increase of appointments that are kept or cancelled in advance but does not counteract the effect of social deprivation on attendance.

Table 1

\begin{tabular}{lccc}
\hline & Reminder & No reminder & NNT (95\% Cl) \\
\hline Number & 115 & 116 & \\
Attended & 84 & 75 & $12(5-29)$ \\
Attended or cancelled & 94 & 89 & $20(6-18)$ \\
\hline
\end{tabular}

Address for correspondence: Prof. Kevin O'Brien, Department of Dental Medicine and Surgery, University Dental Hospital of Manchester, Higher Cambridge Street, Manchester M15 6FH, UK. E-mail: Kevin.O'Brien@man.ac.uk

\section{Commentary}

This interesting and well conducted randomised controlled trial looks at a topic that bedevils most health care providers, that of the failed attendance. Failed attendance leads to the use of strategies such as overbooking to counteract this well known phenomenon. This can lead to increased frustration both in terms of patients having to wait when all booked patients attend. The consequential increased pressure on staff could affect overall quality or care. Acceptance of failed attendance leads to longer waiting lists, so strategies that improve the effective use of clinical time merit proper investigation.

The main finding of improved attendance by those receiving postal prompts is similar to other studies in dentistry and other areas. Calculation of the numbers needed to treat reveal that reminders need to be sent to 12 patients in order to get one less failure. It is interesting to note that the reminders may also be of use in identifying those most likely to fail appointments, for while $23 \%$ of patient in the no reminder group failed to attend $36 \%$ of those not returning their confirmation did not attend as opposed to only a $12 \%$ failure of those reminded patients who returned their confirmation cards.

This study sheds some light on methods which might be used to improve attendance at orthodontic clinics. However, other forms of reminders have been tried most notably telephone reminders and email may be of value into the future. The cost of failed outpatient appointments to the NHS has been estimated at around $£ 280$ million pounds per year so strategies to reduce this are welcomed. The reasons for non-attendance are complex and more detailed work is required looking at for example, the effect of waiting lists, reason for referral, geographical and temporal access to the clinic and the other particular socio-demographic factors of social deprivation.

While this study does confirm an effect of postal reminders more work is required to compare this with other strategies and some analysis of their cost-effectiveness is also required.

\section{Practice point}

- Postal reminders can improve attendance but more research is required into a range of effective strategies for improving outpatient attendance.

\section{David Thomas \\ Regional Director of Postgraduate Dental Education, and Consultant in Dental Public Health, Oxford, UK}

Evidence-Based Dentistry (2004) 5, 14.

doi:10.1038/sj.ebd.6400244 